Letter

\section{Association Between Glandular Infiltrate and Leukopenia in Sjögren Syndrome (SS): Data From the Italian Research Group on SS (GRISS)}

\section{To the Editor:}

We read with great interest the paper by Sharma, et al ${ }^{1}$, who analyzed a cohort of patients with primary Sjögren syndrome ( $\mathrm{pSS}$ ), evaluating differences between a subgroup of subjects with positive minor salivary gland (MSG) biopsy compared to patients with a negative MSG biopsy. The authors found a higher prevalence of anti-La positivity and increased levels of $\operatorname{IgG}$ in the former group and identified a significant upregulation of type I interferon (IFN)-regulated genes in a sample group of patients with positive MSG biopsy.

These interesting data prompted us to analyze serological and immunological features in the same 2 groups in our very large cohort of 1706 pSS patients recruited in the multicenter GRISS (Italian research group on SS) study. To be able to compare the data with those by Sharma, et al ${ }^{1}$, we included only patients with positive anti-Ro antibodies who underwent MSG biopsy and compared the subjects with a positive result, defined as a focus score (FS) $\geq 1$, to patients with negative biopsy (FS $<1$ ). In accordance with both the American-European Consensus Group and the American College of Rheumatology/European League Against Rheumatism classification criteria ${ }^{2,3}$, all patients with $\mathrm{FS}<1$ were defined as negative in our cohort, so a separate analysis of patients with FS between 0 and 1 was not possible. However, the subjects included in the group by Sharma, et al $l^{1}$ represented a small minority (5\%) with apparent negligible effect on the reported results. For statistical analysis, we performed a Yates chi-square test or a Fisher exact test as appropriate and chose an $\alpha$ of 0.05 for significance. To account for type 1 error, we performed a Bonferroni correction and considered data significant for $P \leq 0.01$.

A total of 638 ( $95 \%$ female) subjects with a mean age at diagnosis of 50 \pm 14 years and a mean disease duration of $7 \pm 7$ years were analyzed. Age, sex, and disease duration did not differ between the 2 groups. As shown in Table 1, we found no difference in the prevalence of a number of serological variables, including hypergammaglobulinemia. Although we observed a nonsignificant trend toward a higher percentage of patients with circulating rheumatoid factor and possible expression of B cell hyperreactivity within the group with positive histological findings, we could not compare the data on hyper IgG due to the lack of specific data on Ig isotypes in our cohort. Interestingly, however, patients with a positive MSG biopsy had a significant higher prevalence of leukopenia in comparison to the negative MSG biopsy group.

Although usually mild, leukopenia is a common feature in patients with pSS. There is robust evidence linking leukopenia to a more severe glandular

Table 1. Serological characteristics of $\mathrm{pSS}$ patients with or without a positive MSG biopsy.

Characteristic

$\begin{array}{cc}\text { Positive MSG } & \text { Negative MSG } \\ \text { Biopsy, } \mathrm{n}=600 & P \\ \text { Biopsy, } \mathrm{n}=38\end{array}$

$$
\text { Biopsy, } \mathrm{n}=600 \quad \text { Biopsy, } \mathrm{n}=38
$$

\begin{tabular}{lccc}
\hline Anti-La+ & $281(46.8)$ & $14(36.8)$ & 0.303 \\
Hypergammaglobulinaemia & $356(59.3)$ & $23(60.5)$ & 1.000 \\
Hypocomplementaemia & $134(22.3)$ & $6(15.8)$ & 0.457 \\
Leukopenia & $178(29.7)$ & $4(10.5)$ & $\mathbf{0 . 0 0 9}$ \\
Rheumatoid factor & $372(62.0)$ & $16(42.1)$ & 0.024 \\
\hline
\end{tabular}

Values are $\mathrm{n}(\%)$ unless otherwise specified. Differences are considered significant for $\mathrm{p} \leq 0.001$ (bold face). MSG: minor salivary gland; $\mathrm{pSS}$ : primary Sjögren syndrome. involvement, especially in patients with lymphoma or prelymphomatous conditions $s^{4,5}$. Intriguingly, these data appear to fit with the increased type I IFN signature observed by Sharma, et al ${ }^{1}$ in MSG biopsy-positive patients. Indeed, a clear association between increased IFN signature, leukopenia, and higher FS has been demonstrated ${ }^{6,7}$. The mechanisms linking leukopenia, IFN signature, and glandular infiltration are not fully elucidated. It has been suggested that type I IFN is implicated in a positive feedback loop involving B cells, T cells, and neutrophils, stimulating increased Fas-mediated apoptosis, and in turn, further type I IFN secretion and signaling. An association has also been demonstrated between single-nucleotide polymorphisms of signal transducer and activator of transcription 4 (STAT4), a downstream effector of IFN-signaling pathways, and clinical features of pSS such as leukopenia?

Some of the possible explanations of the different results obtained may be the higher number of subjects evaluated in our analysis (638 vs 229) and the rate of positive MSG biopsy that is significantly lower in the study by Sharma, et al ${ }^{1}$ (73\%; vs $94 \%$ in our cohort) ${ }^{2,3}$. This may be due to our low propensity to diagnose pSS in patients with negative MSG biopsy who have very mild, unspecific, or nontypical clinical manifestations; it may also be caused by high prevalence of non-SS-related sicca symptoms in the otherwise healthy general population with isolated anti-Ro positivity. In this context, it is an important reminder that when highly sensitive assays are employed, the detection of circulating isolated low-titer anti-Ro antibodies in the general population is rather common and could lead to potential overdiagnosis of $\mathrm{pSS}$ if classification criteria are applied.

In conclusion, we believe that our data provide an interesting integration of Sharma, et als results' ${ }^{1}$ In fact, the association of leukopenia with glandular infiltrate and IFN signature may represent an interesting loop, which suggests a close pathogenic relationship that deserves further investigations.

Giacomo Cafaro ${ }^{1}$ (D), MD

Roberto Gerli ${ }^{1}$, MD

Elena Bartoloni ${ }^{1}$ D, MD

${ }^{1}$ Rheumatology Unit, Department of Medicine, University of Perugia, Perugia, Italy.

Address correspondence to Prof. R. Gerli, Rheumatology Unit, Department of Medicine, University of Perugia, P.le Menghini 1, 06129 Perugia, Italy. Email: roberto.gerli@unipg.it.

\section{REFERENCES}

1. Sharma R, Chaudhari KS, Kurien BT, Grundahl K, Radfar L, Lewis DM, et al. Sjögren syndrome without focal lymphocytic infiltration of the salivary glands. J Rheumatol 2020;47:394-9.

2. Shiboski CH, Shiboski SC, Seror R, Criswell LA, Labetoulle M, Lietman TM, et al; International Sjögren's Syndrome Criteria Working Group. 2016 American College of Rheumatology/ European League Against Rheumatism classification criteria for primary Sjögren's syndrome: a consensus and data-driven methodology involving three international patient cohorts. Arthritis Rheumatol 2017;69:35-45.

3. Vitali C, Bombardieri S, Jonsson R, Moutsopoulos HM, Alexander EL, Carsons SE, et al; European Study Group on Classification Criteria for Sjögren's Syndrome. Classification criteria for Sjögren's syndrome: a revised version of the European criteria proposed by the American-European Consensus Group. Ann Rheum Dis 2002;61:554-8.

4. Gerli R, Muscat C, Giansanti M, Danieli MG, Sciuto M, Gabrielli A, et al. Quantitative assessment of salivary gland inflammatory infiltration in primary Sjogren's syndrome: its relationship to different demographic clinical and serological features of the disorder. Br J Rheumatol 1997;36:969-75.

5. Quartuccio L, Isola M, Baldini C, Priori R, Bartoloni Bocci E,

Personal non-commercial use only. The Journal of Rheumatology Copyright $\subset$ $\subset$ 2020. All rights reserved. 
Carubbi F, et al. Biomarkers of lymphoma in Sjögren's syndrome and evaluation of the lymphoma risk in prelymphomatous conditions: results of a multicenter study. J Autoimmun 2014;51:75-80.

6. Hall JC, Baer AN, Shah AA, Criswell LA, Shiboski CH, Rosen A, et al. Molecular subsetting of interferon pathways in Sjögren's syndrome. Arthritis Rheumatol 2015;67:2437-46.

7. Bodewes IL, Al-Ali S, van Helden-Meeuwsen CG, Maria NI, Tarn J, Lendrem DW, et al; Systemic interferon type I and type II signatures in primary Sjögren's syndrome reveal differences in biological disease activity. Rheumatology 2018;57:921-30.

8. Brkic Z, Versnel MA. Type I IFN signature in primary Sjögren's syndrome patients. Expert Rev Clin Immunol 2014;10:457-67.

9. Colafrancesco S, Ciccacci C, Priori R, Latini A, Picarelli G, Arienzo F, et al. STAT4, TRAF3IP2, IL10, and HCP5 polymorphisms in Sjögren's syndrome: association with disease susceptibility and clinical aspects. J Immunol Res 2019;2019:7682827. 